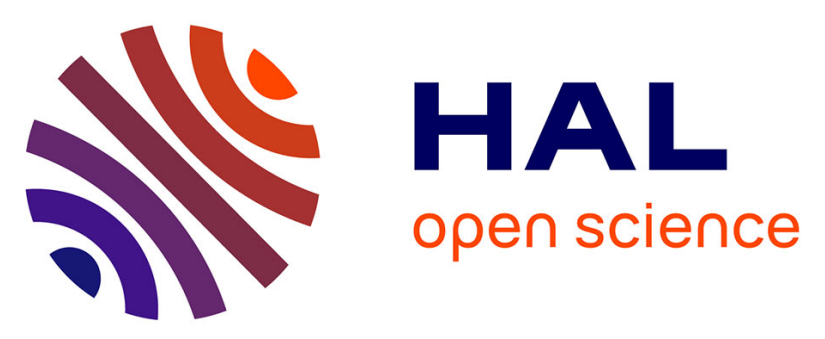

\title{
Compressed sensing subtracted rotational angiography with multiple sparse penalty
}

Hélène Langet, C. Riddell, Y. Trousset, Arthur Tenenhaus, Elisabeth Lahalle, Gilles Fleury, Nikos Paragios

\section{To cite this version:}

Hélène Langet, C. Riddell, Y. Trousset, Arthur Tenenhaus, Elisabeth Lahalle, et al.. Compressed sensing subtracted rotational angiography with multiple sparse penalty. 2012 9th IEEE International Symposium on Biomedical Imaging (ISBI 2012) , May 2012, Barcelone, Spain. pp.884-887, 10.1109/ISBI.2012.6235690 . hal-00725950

\section{HAL Id: hal-00725950 \\ https://hal-centralesupelec.archives-ouvertes.fr/hal-00725950}

Submitted on 28 Aug 2012

HAL is a multi-disciplinary open access archive for the deposit and dissemination of scientific research documents, whether they are published or not. The documents may come from teaching and research institutions in France or abroad, or from public or private research centers.
L'archive ouverte pluridisciplinaire HAL, est destinée au dépôt et à la diffusion de documents scientifiques de niveau recherche, publiés ou non, émanant des établissements d'enseignement et de recherche français ou étrangers, des laboratoires publics ou privés. 


\title{
COMPRESSED SENSING SUBTRACTED ROTATIONAL ANGIOGRAPHY WITH MULTIPLE SPARSE PENALTY
}

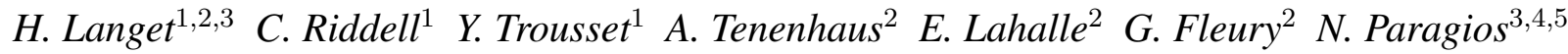 \\ ${ }^{1}$ GE Healthcare, Buc, France \\ ${ }^{2}$ Supélec, Department of Signal Processing and Electronic Systems, Gif-sur-Yvette, France \\ ${ }^{3}$ Ecole Centrale Paris, Center for Visual Computing, Châtenay-Malabry, France \\ ${ }^{4}$ Ecole des Ponts ParisTech, Center for Visual Computing, Champs-sur-Marne, France \\ ${ }^{5}$ INRIA Saclay, GALEN team, Orsay, France
}

\begin{abstract}
Digital Subtraction Rotational Angiography (DSRA) is a clinical protocol that allows three-dimensional (3D) visualization of vasculature during minimally invasive procedures. $\mathrm{C}$-arm systems that are used to generate $3 \mathrm{D}$ reconstructions in interventional radiology have limited sampling rate and thus, contrast resolution. To address this particular subsampling problem, we propose a novel iterative reconstruction algorithm based on compressed sensing. To this purpose, we exploit both spatial and temporal sparsity of DSRA. For computational efficiency, we use a proximal implementation that accommodates multiple $\ell_{1}$-penalties. Experiments on both simulated and clinical data confirm the relevance of our strategy for reducing subsampling streak artifacts.
\end{abstract}

Index Terms - Rotational angiography, Compressed sensing, X-ray tomography, Iterative algorithms, Sparsity, Proximal operators

\section{INTRODUCTION}

Compressed sensing provides a new framework for signal recovery, which under certain assumption could surpass the fundamental Shannon-Nyquist sampling limit [1]. Threedimensional interventional radiology faces sampling issues, since C-arm technology is currently limited by coarse angular sampling. Rotation speed of the device must be set as high as safely possible to minimize the acquisition (scan) duration, while the detector acquisition frame rate limits the total number of views. We investigate the sampling problem in a well-defined clinical practice called DSRA, which consists in performing two scans in a single protocol: a mask scan, which is acquired without injection, and a contrast scan, which is acquired after injecting vessels with contrast medium. Subtraction of the mask from the contrast enhances vasculature visualization in presence of dense structures such as bones. The sampling limitations of $\mathrm{C}$-arm systems give rise to typical subsampling structures known as streak artifacts that degrade the overall quality of all three reconstructed volumes. In particular, whereas subsampling has finally little incidence on the visualization of highly contrasted structures, streak artifacts generated by these latter might hide weakly contrasted structures such as soft tissues, thus limiting the usability of the technique.

Sparsity is modeled with $\ell_{1}$-norms, whose minimization under a data constraint generates sparse signal approximations. An extension of the iterative filtered backprojection (iFBP) that deals with sparse penalties was introduced in [2]. Several works, among which [3] and references therein, have focused on the reconstruction of piecewise constant approximations of more clinically relevant type of objects, showing streak artifact reduction, but at the expense of an overall change in the image appearance, that may not be clinically acceptable. In the DSRA case, sparsity assumptions on the subtracted volume - which seems realistic since vessels are naturally sparse - promotes redundancy of the nonopacified structures captured in both scans. Such an approach eliminates the need for identical sampling of the mask and the contrast scans, and therefore makes possible to double the sampling of the nonopacified structures by adopting scans that sample two sets of equiangular-spaced interleaved positions as illustrated in Fig.1. In the non-subtracted case, it is possible to reduce streaks of sparse structures over a non-sparse background by progressively relaxing a sparse constraint on the solution as demonstrated in [4].

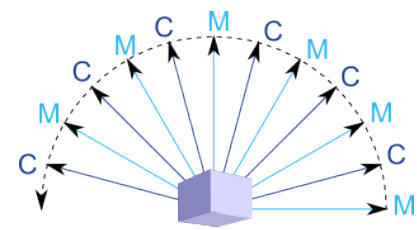

Fig. 1. Contrast and mask scans sample two sets of equiangular-spaced interleaved positions.

We propose in the following a novel multiple penalty for DSRA reconstruction, that follows the compressed sensing 
theory and relies on the three previously cited approaches [24]. This strategy requires to generate a solution that satisfies several priors simultaneously and thus minimize a functional including multiple $\ell_{1}$-terms. The next section presents the application of proximal splitting to the reconstruction problem with a sparse multiple penalty dedicated to DSRA. The relevance of our approach is then evaluated in parallel geometry on simulated data and in cone-beam geometry on real clinical data. We discuss remaining limitations and perspectives of our work in the final section.

\section{METHODOLOGY}

It has been shown in [1] that perfect reconstruction of a piecewise constant image is achievable through minimization of its total variation (TV). These results have highlighted the interest of casting the reconstruction problem as an optimization problem constrained with a suitable sparse penalization.

\section{1. $\ell_{1}$-penalized reconstruction}

We define $f=\left(f_{C}, f_{M}\right)^{T}$ as the vector containing respectively the contrast volume and the mask volume, $p=$ $\left(p_{C}, p_{M}\right)^{T}$ as the vector containing respectively the injected projections and the mask projections, and $R=\operatorname{diag}\left\{R_{C}, R_{M}\right\}$ as the block-diagonal matrix describing the trajectory of respectively the contrast and the mask scans.

Let $Q$ be the quadratic term that reflects the data acquisition process $R f=p$. Here, we define $Q$ as the distance between $p$ and projections of $f$ weighted by $W: Q(f)=$ $\frac{1}{2}\|W(R f-p)\|_{2}^{2}$. The introduction of $W$ allows the integration of statistical information or of a filtering step [5] that boosts convergence if required. For computational efficiency, we choose $W$ such that $W^{2}=D$, where $D$ is the ramp filter in the Fourier domain. The minimization of the corresponding expression of $Q$ is referred to as iFBP. Let $\chi$ be a sparsity penalty with weight $\alpha>0$. We consider the following constrained reconstruction problem:

$$
\underset{f}{\operatorname{argmin}} Q(f)+\alpha \chi(\Phi f)
$$

where $\Phi$ is a linear operator that can be for instance gradient (TV-penalization) or wavelet transform. Proximal splitting methods [6] allows for building a sequence that converges to to the minimum of eq.1. As it was done in [2], we use the forward-backward scheme given below:

$$
f^{(n+1)}=\operatorname{prox}_{\tau \alpha \chi}\left(f^{(n)}-\tau \nabla Q\left(f^{(n)}\right)\right)
$$

where the proximal operator denoted $\operatorname{prox}_{\tau \alpha \chi} u$ is the unique minimum of $\tau \alpha \chi(\cdot)+\frac{1}{2}\|\cdot-u\|_{2}^{2}$ and parameter $\tau>0$ corresponds to the gradient descent step. Proximal operators generalize the class of projectors onto convex sets to include standard image processing tools such as TV filtering and wavelet filters with soft-thresholding.

\subsection{Compressed sensing reconstruction}

In the subsampling case, there are many minimizers of the quadratic term and compressed sensing enables to identify the solution as the sparsest minimizer. This approach is distinct from regularization, which consists in finding the best tradeoff between the fitting term and a given sparse penalty. However, it is possible to perform compressed sensing reconstruction by defining a set of weights: $A=\left\{\alpha_{n} \mid n=1, \cdots, N\right\}$, such that the regularization strength is decreased at each iteration: $\alpha_{1} \geq \cdots \geq \alpha_{N}=0$, where $N$ is the total number of iterations. This strategy is known as "continuation" or "homotopy" in the convex optimization community [7]. It allowed for processing sparse structures over a non-sparse background when used with soft-background subtraction (SBS) operator [4].

\subsection{Multiple sparse penalty dedicated to DSRA}

We propose to constrain DSRA reconstruction with a sparse penalty $\chi(f)$ that is expressed as the combination of:

- a temporal component $\varphi_{t}(f)$ : to mix background information from the contrast and the mask without losing vessel quantification, we apply the one-dimensional (1D) Haar wavelet transform $H_{t}$ to isolate the temporal component. In the case of DSRA, it simply captures the vascular structures that are filled by the contrast and contained in the subtracted volume $f_{S}=f_{C}-f_{M}$;

- a spatial component $\varphi_{x y z}(f)$ : to remove streaks from the contrast volume and prevent the transfer of vesselrelated information (including streaks) in the mask, we penalize both the mask and the contrast volumes 11norms.

Moreover, positivity of all three volumes $f_{M}, f_{C}$ and $f_{S}$ is ensured with the indicator function $\iota_{+}(\cdot)$. Overall, the DSRA reconstruction problem is constrained with the following multiple sparse penalty:

$$
\left\{\begin{aligned}
\chi(f) & =\varphi_{x y z}(f)+\varphi_{t}(f) \\
\varphi_{x y z}(f) & =\alpha_{x y z}\|f\|_{1}+\iota_{+}(f) \\
\varphi_{t}(f) & =\alpha_{t}\left\|H_{t} f\right\|_{1}+\iota_{+}\left(H_{t} f\right)
\end{aligned}\right.
$$

where $\alpha_{x y z}$ and $\alpha_{t}$ are regularization parameters such that $\alpha_{x y z}$ is linearly decreased to zero during the minimization process, while $\alpha_{t}$ is set to a fixed value. The proximal operator that is associated to the spatial term $\varphi_{x y z}(f)$ (respectively, the temporal term $\varphi_{t}(f)$ ) is simply the SBS operator with threshold $\alpha_{x y z} \tau$ (respectively $\alpha_{t} \tau$ ). The computation of the proximal operator associated to a multiple sparsity constraint of the form $\chi(f)=\sum_{k=1}^{K} \varphi_{k}(f)$ is handled by the Dykstralike proximal algorithm [6]. An overview of its parallel implementation in pseudocode is given hereafter. The computation of $\operatorname{prox}_{\tau \chi}$ is integrated in the forward-backward algorithm given in eq. 2 by setting input $u=f^{(n)}-\tau \nabla Q\left(f^{(n)}\right)$ and output $f^{(n+1)}=v^{(M+1)}$ : 


$$
\begin{aligned}
& \text { Set } v^{(1)}=u \text { and } h_{1}^{(1)}=\cdots=h_{k}^{(1)}=v^{(1)} \\
& \text { for } m \leftarrow 1 \text { to } M \text { do } \\
& \text { for } k \leftarrow 1 \text { to } K \text { do } \\
& \qquad g_{k}^{(m)}=\operatorname{prox}_{\tau \varphi_{k}} h_{k}^{(m)} \\
& v^{(m+1)}=\sum_{k=1}^{m} g_{k}^{(m)} \\
& \text { for } k \leftarrow 1 \text { to } K \text { do } \\
& \left\lfloor h_{k}^{(m+1)}=v^{(m+1)}+h_{k}^{(m)}-g_{k}^{(m)}\right.
\end{aligned}
$$

Algorithm 1: Parallel Dykstra-like proximal algorithm scheme

\section{RESULTS}

We evaluated our approach on both simulated and clinical data. Intensities are given in positive Hounsfield Unit (HU), i.e. air is 0 instead of $-1000 \mathrm{HU}$. Reconstruction settings are as follows: iFBP algorithm was used with $\mathrm{N}=20$ iterations and a gradient step $\tau=1$. Note that the computation cost of an iFBP iteration is about twice that of FBP. For decreasing SBS, $\alpha_{x y z}^{(n)}$ was initialized at $\alpha_{x y z}^{(1)}$ equal to $95 \%$ of the maximum value of the filtered backprojection (FBP) reconstruction and linearly decreased to $\alpha_{x y z}^{(N)}=0$.

For our simulation, we used a $512 \times 512$ cerebral CT cross-section as mask image. We simulated the contrast image by adding to the mask synthetic disks that represent opacified arteries. The value of the simulated injected vessels varies from 2000 to $3000 \mathrm{HU}$, while soft tissue values (around $1000 \mathrm{HU}$ ) and bone values (around $2000 \mathrm{HU}$ ) are those of the original CT slice. We produced interleaved mask and contrast scans in parallel geometry with settings that fit DSRA routine, in which the $\mathrm{C}$-arm system records projections at 30 frames/s during an approximately $200^{\circ}$ rotation at $40 \%$ delivering 150 views in total. We compare the reconstruction quality of the background structures in Fig.2. Root mean square deviation (RMSD) between the reconstruction and the true mask image $\tilde{f}$ over the $J_{b}$ pixels of the background structures are also given to appreciate image quality in a quantitative manner: $d=\sqrt{\frac{1}{J_{b}} \sum_{j=1}^{J_{b}}\left(f_{j}-\tilde{f}_{j}\right)^{2}}$. Standard reconstruction of the mask with a double sampling (300 views) is shown in Fig.2a as reference: its level of streaks is the lowest level that can be achieved with the approach we developed $(d=9 \mathrm{HU})$. Subsampled standard reconstruction (150 views) yields streak artifacts that, in particular, makes cerebral sulci visualization difficult, as shown in Fig.2b. This degradation was confirmed by an increased RMSD value $d=25 \mathrm{HU}$. The contrast image (see Fig.2c) presents additional streaks due to the injected vessels. Figure $2 \mathrm{~d}$ displays mask reconstruction penalized by the temporal constraint $\varphi_{t}(f)$ only, with weight $\alpha_{t}=5$, which was the reconstruction method proposed by [2]. It allows for removing the background streaks and for recov- ering a background resolution similar to the reference one. Nevertheless, we notice that a small amount of the injected vessel intensity is transferred from the contrast to the mask (vessel marks). Vessel mark streaks affect the background similarly to vessel streaks - the deterioration is less important though $(d=15 \mathrm{HU})$. Streaks are especially visible near the skull bone on the right side of the image. Vessel marks and streaks are not visible anymore in Fig.2e that was produced with the multiple penalty $\chi(f)$. We measured $d=11 \mathrm{HU}$, which confirms the actual image quality improvement. True vessel intensity is recovered, which would not be the case if we simply computed the average reconstruction of the mask and the contrast volume. For all reconstructions, sparsity of the subtracted volume (not shown here) is preserved. We used $\alpha_{t}=0$ rather than a low threshold to avoid both bias and vessel transfer from the contrast to the mask.

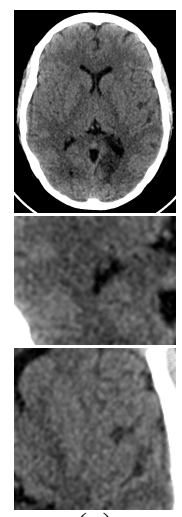

(a)

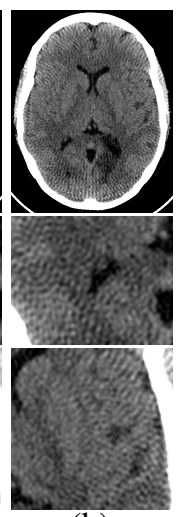

(b)

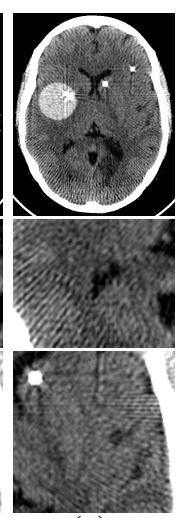

(c)

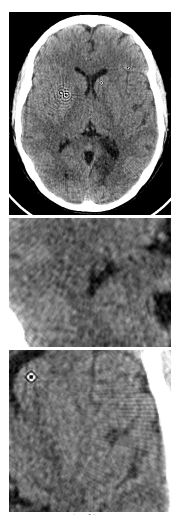

(d)

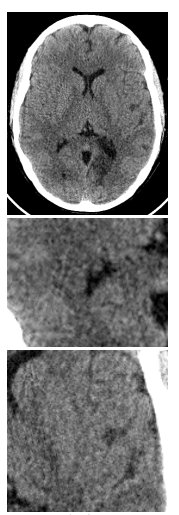

(e)
Fig. 2. DSRA reconstruction from simulated data (HU range: 1020 to 1100). (a) Mask iFBP reconstruction with double sampling (300 views). (b) Mask iFBP reconstruction. (c) Contrast iFBP reconstruction. (d) Mask iFBP reconstruction penalized by $\varphi_{t}(f)$ only, with $\alpha_{t}=5$. (e) Mask iFBP reconstruction penalized by $\chi(f)$ with $\alpha_{t}=0$

We also evaluated the reconstruction quality of the proposed algorithm on clinical data acquired on an Innova 3100 C-arm system (GE Healthcare, Chalfont St. Giles, UK). We now face the additional challenges of 3D cone-beam geometry, short-scan acquisition and truncated subsampled data. Since there exists no clinical protocols with interleaved scans, we built an interleaved acquisition pattern with 75 views for each scan by taking one view every two views with a shift of one view when starting the contrast scan as illustrated in Fig.1. Iterative FBP dedicated to cone-beam geometry relies on the Feldkamp approximated inversion (FDK) and is referred to as iterative Feldkamp (iFDK). For all reconstructions, a volume of $320^{3}$ voxels was computed. Figure $3 \mathrm{com}-$ pares standard reconstruction (iFDK with a positivity constraint, displayed in the first line) and iFDK with the multiple sparse penalty $\chi(f)$ (displayed in second line). In agreement with the simulated results, our algorithm yields significantly 
less streaks than standard reconstruction. The resulting resolution improvement is best seen in the petrous part of the left temporal bone (right side of the slice, detail zoomed in Fig.3b) that contains the inner ear: thin details such as tympanic cavity, canals, and sutures are more accurate. The observations are confirmed when computing the mean and standard deviation in a region of interest of 900 voxels within soft tissues, which gives $1308 \pm 243 \mathrm{HU}$ for the standard reconstruction and $1077 \pm 166 \mathrm{HU}$ for our reconstruction. This corresponds to a $20 \%$ Signal-to-Noise-Ratio (SNR) increase (6.5 vs. 5.4). Note that an SNR value of $10.0(1116 \pm 112 \mathrm{HU})$ is found for the standard reconstruction with 150 views (not shown here). Looking at the subtracted volume of Fig.3c, we notice that even if our approach yields sparser structures than standard reconstruction, the whole volume is not very sparse, which limits the extension of the background structures where mask and contrast volumes can be mixed and sampling improved.

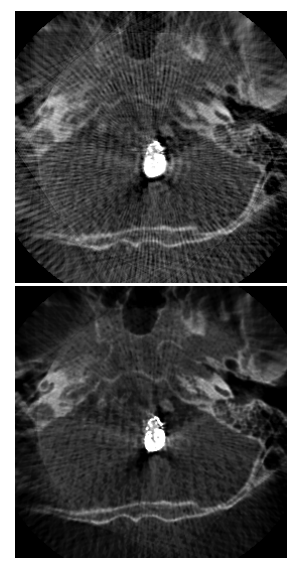

(a)

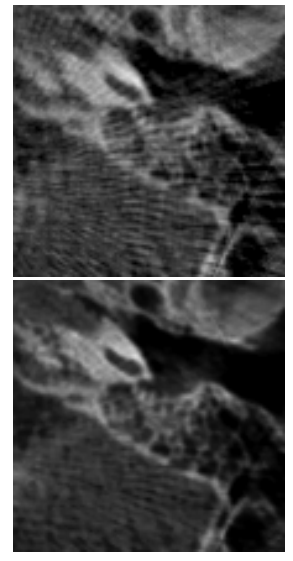

(b)

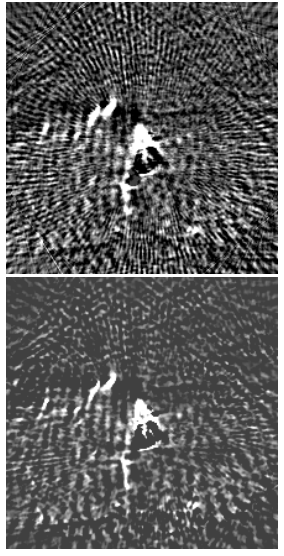

(c)
Fig. 3. DSRA reconstruction from clinical data. First line: Mask iFDK reconstruction with spatial positivity constraint. Second line: Mask iFDK reconstruction penalized by $\chi(f)$ with $\alpha_{t}=0$. (a) Axial slice (HU range: 250 to 5350). (b) Detail of the petrous part of the left temporal bone in (a). (c) Subtracted slice (HU range: -500 to 1500 ).

\section{DISCUSSION AND CONCLUSION}

We proposed a novel multiple sparsity constraint that promotes both spatial and temporal sparsity of the data - by using the $\ell_{1}$-penalized iFBP scheme - for performing compressed sensing DSRA reconstruction. Our implementation relies on proximal splitting methods, which has proven to be a very efficient strategy for $\ell_{1}$-penalized minimization. The results that we obtained on both simulated and clinical data showed that our approach outperforms standard reconstruction in terms of background restoration and streak removal. Unlike reconstruction regularized by Total Variation (TV), which has been proven to be an efficient method to get rid of the streak artifacts and converges to a piecewise-constant approximation of the solution, our approach does not change image appearance. Moreover, this quality improvement is performed at a reasonable cost of 20 iterations and a small change in the acquisition protocol so that both scans sample interleaved angular positions. The small amount of iterations required to reach convergence let us think that it could be realistic to use such iterative algorithm in clinical practice.

\section{REFERENCES}

[1] E. Candès, J. Romberg, and T. Tao, "Robust Uncertainty Principles: Exact Signal Reconstruction from Highly Incomplete Frequency Information," IEEE Tr. Information Theory, vol. 52, no. 2, pp. 489-509, Feb. 2006.

[2] H. Langet, C. Riddell, Y. Trousset, E. Lahalle, A. Tenenhaus, G. Fleury, and N. Paragios, "Sparsity Constraints and Dedicated Acquisition Protocols for Improved Digital Subtraction Rotational Angiography," in Fully3D, 2011.

[3] Ludwig Ritschl, Frank Bergner, Christof Fleischmann, and Marc Kachelrieß, "Improved Total Variation-Based CT Image Reconstruction Applied to Clinical Data," Physics in Medicine and Biology, vol. 56, no. 6, pp. 1545-1561, Mar. 2011.

[4] H. Langet, C. Riddell, Y. Trousset, A. Tenenhaus, E. Lahalle, G. Fleury, and N. Paragios, "Compressed Sensing Based 3D Tomographic Reconstruction for Rotational Angiography," in MICCAI, vol. 6891, pp. 97-104. 2011.

[5] C. Riddell, A. Savi, M.C. Gilardi, and F. Fazio, "Frequency Weighted Least Squares Reconstruction of Truncated Transmission SPECT Data," IEEE Tr. Nuclear Science, vol. 43, no. 4, pp. 2292-2298, Aug. 1996.

[6] P. L. Combettes and J.-C. Pesquet, "Proximal Splitting Methods in Signal Processing," in Fixed-Point Algorithms for Inverse Problems in Science and Engineering, vol. 49, chapter 10, pp. 185-212. 2011.

[7] B. Efron, T. Hastie, I. Johnstone, and R. Tibshirani, "Least angle regression," Annals of Statistics, vol. 32, pp. 407-499, 2004. 